\title{
F10, a novel hydatidiform mole-associated gene, inhibits the paclitaxel sensitivity of A549 lung cancer cells by downregulating BAX and caspase-3
}

\author{
YALI SONG ${ }^{1 *}$, WEI CAO $^{2 *}, \mathrm{XI} \mathrm{ZHU}^{1}$, ZHUOLIN QIU $^{1}$, XIAOPING YANG $^{1}$, \\ JING LIU ${ }^{1}$, RUOTING XU ${ }^{3}$, WEIZHUANG YUAN ${ }^{3}$ and SONG QUAN ${ }^{1}$ \\ Departments of ${ }^{1}$ Obstetrics and Gynecology, ${ }^{2}$ Nephrology and ${ }^{3}$ Clinical Medicine, \\ Nanfang Hospital, Southern Medical University, Guangzhou, Guangdong 510515, P.R. China
}

Received July 26, 2015; Accepted November 17, 2016

DOI: $10.3892 / \mathrm{ol} .2017 .5749$

\begin{abstract}
F10 is a novel hydatidiform mole (HM)-associated gene that was initially identified during a study into the pathogenesis of HMs. However, the role of the F10 gene requires further investigation. Our, previous studies have indicated that F10 may be involved in the malignant transformation of HMs and the development of certain types of adenocarcinoma, and that the overexpression of F10 may lead to excessive proliferation and decreased apoptosis of A549 cells. The present study aimed to investigate whether F10 may suppress the sensitivity of A549 lung cancer cells to paclitaxel therapy. A previously established F10-overexpressing A549 cell line (A549-F10) was treated with paclitaxel, using untransfected A549 cells and A549-mock cells (non-carrier A549) as the controls. These three groups of cells were subsequently examined by an MTT cell proliferation assay and a TUNEL-fluorescein isothiocyanate/Hoechst 33258 apoptosis assay. A western blot analysis was used to determine the expression levels of the pro-apoptotic genes B-cell lymphoma-2-associated X protein (BAX) and caspase-3. The effects of paclitaxel treatment on the proliferation and apoptosis of A549 cells were compared between the aforementioned cell lines. It was revealed that F10 inhibited the chemosensitivity of A549 cells to paclitaxel, as demonstrated by the decreased rates of growth inhibition and apoptosis in the A549-F10 group compared with the two control groups. Furthermore, the A549-F10 cells treated with
\end{abstract}

Correspondence to: Dr Song Quan or Dr Yali Song, Department of Obstetrics and Gynecology, Nanfang Hospital, Southern Medical University, 1838 North Guangzhou Avenue, Guangzhou, Guangdong 510515, P.R. China

E-mail: quansong@smu.edu.cn

E-mail: sy1950446@126.com

*Contributed equally

Key words: F10, A549 cells, chemosensitivity, B-cell lymphoma-2 (BCL-2)-associated X protein, caspase-3 paclitaxel exhibited significantly lower expression levels of the pro-apoptotic genes. The results of the current study demonstrate that F10 may inhibit the chemosensitivity of A549 cells to paclitaxel and that this inhibitory effect may be mediated by the downregulation of BAX and caspase-3 expression, which subsequently inhibits cell apoptosis.

\section{Introduction}

Chemotherapy is an established therapeutic approach for human malignancies. It has been well established that chemotherapeutic agents can damage tumor cells by inducing cell apoptosis (1). Paclitaxel is one of the most efficacious chemotherapy drugs that exerts antitumor effects through the induction of cell apoptosis (2-4), and the therapeutic value of paclitaxel has previously been demonstrated in studies of lung cancer (5-7). However, the development of resistance to chemotherapy frequently presents a challenge to the successful treatment (8). Therefore, the identification of the molecular mechanisms underlying chemosensitivity may facilitate the improvement of current treatment strategies.

F10 is a novel hydatidiform mole (HM)-associated gene that was identified in our previous study on the pathogenesis of HMs (9); a suppression subtractive hybridization technique was used and the novel gene F10 was screened from the cDNA libraries established between HM tissues and the normal villi of early pregnancies (GenBank no. AB196290). However, the function of this gene remained undetermined. Since the gene was kept in the F10 location of 96-well plates, it was termed the F10 gene.

Our previous study confirmed that the expression of the F10 gene was positive in HMs, invasive moles and choriocarcinomas, and that its expression intensity increased gradually in these conditions (10). Furthermore, the results indicated that the expression of the F10 gene was positive in a variety of adenocarcinomas, including ovarian cancer, endometrial cancer, breast cancer, liver cancer and gastric cancer (11). However, it was negative in certain normal tissues, including endometrial and cervical epithelium (11). These results suggested that the F10 gene may have a role in the malignant transformation of HMs and in the incidence of certain types of adenocarcinoma. 
The overexpression of F10 in A549 human lung cancer cells was demonstrated to promote cell proliferation by increasing the expression of proliferating cell nuclear antigen and cyclin D1 (12), and to inhibit cell apoptosis via the downregulation of the pro-apoptotic genes B-cell lymphoma-2 (BCL-2)-associated X protein (BAX) and caspase-3 (13). A previous study revealed that F10 may upregulate the expression of BCL-2, which may enhance the tumorigenicity of the A549 lung cancer cell line in nude mice (14). Thus, F10 may facilitate tumorigenesis and cancer progression by promoting cell proliferation and inhibiting cell apoptosis.

However, few studies have been conducted concerning the biological functions of F10 in lung cancer, particularly in drug resistance (15). Therefore, the present study was conducted to examine the hypothesis that F10 may have an important role in the chemoresistance of cancer cells. The function of F10 in the drug-resistance of A549 lung cancer cells was examined, and the underlying mechanisms of F10 with regard to this chemosensitivity were investigated through the examination of BAX and caspase- 3 expression.

\section{Materials and methods}

Materials. Paclitaxel $(6 \mathrm{mg} / \mathrm{ml})$ was obtained from Shenzhen Neptunus Interlong Bio-Technique Co., Ltd. (Shenzhen, China). The mouse monoclonal anti-human BAX (cat. no. BA0315) and anti-human $\beta$-actin (cat. no. BM0626) antibodies were purchased from Wuhan Boster Biological Technology, Ltd. (Wuhan, China). The horseradish peroxidase-labeled rabbit anti-mouse monoclonal anti-human caspase-3 secary antibody (cat. no. 9668; clonality, 3G2) was obtained from Cell Signaling Technology, Inc. (Danvers, MA, USA). The rabbit anti-mouse secondary antibody was purchased from Dako (Glostrup, Denmark).

Cell culture and treatment. The A549 human lung cancer cell lines, including the untransfected A549 cells, A549-mock (non-carrier A549) cells and A549-F10 cells (which overexpress F10), were obtained from the laboratory of Southern Medical University, Guangzhou, China, as described previously (13), and cultured in RPMI-1640 medium supplemented with $10 \%$ fetal bovine serum (Hangzhou Sijiqing Biological Engineering Materials Co., Ltd., Hangzhou, China). The cells were cultured in a humidified atmosphere with $5 \% \mathrm{CO}_{2}$ at $37^{\circ} \mathrm{C}$.

The cells were divided into three groups: A549-F10, A549-mock (the negative control group) and untransfected A549. Paclitaxel was added to the culture medium of each cell group prior to the cells being added at a density of $1 \times 10^{4}$ cells $/ 200 \mu \mathrm{l}$ complete medium. The final concentration of paclitaxel was maintained at $10 \mathrm{nmol} / \mathrm{ml}$.

MTT cell proliferation assay. Cell proliferation was determined using an MTT assay (Sigma-Aldrich, St. Louis, MO, USA), according to the manufacturer's protocol. The cells treated with paclitaxel, including untransfected A549, A549-mock and A549-F10 cells, were seeded into a 96-well plate at a density of $1 \times 10^{4}$ cells per $200 \mu \mathrm{l}$ complete medium. The cells were incubated with $5 \% \mathrm{CO} 2$ at $37^{\circ} \mathrm{C}$ for $0.5,1,6,12,24$ or $48 \mathrm{~h}$ (each experiment was conducted in sextuplicate for each time point), following which $20 \mu \mathrm{l}$ of $5 \mathrm{mg} / \mathrm{ml} \mathrm{MTT}$ was added to each well. Following a 4-h incubation with $5 \% \mathrm{CO}_{2}$ at $37^{\circ} \mathrm{C}$, the media was removed and $150 \mu \mathrm{l}$ dimethyl sulfoxide (Sigma-Aldrich) was added to each well to stop the reaction. Following a 5-min mixing period, the optical density $(490 \mathrm{~nm})$ was measured with a Bio-Tek microplate reader (Bio-Tek Instruments, Inc., Winooksi, VT, USA) and a tumor cell growth standard curve was obtained for the assessment of cell proliferation. The rate of growth inhibition was calculated as previously described (16): Growth inhibition $(\%)=\left[\left(1-\mathrm{A}_{48 \mathrm{~h}}\right) /\left(\mathrm{A}_{0 \mathrm{~h}}\right)\right] \times 100$, where A represents the absorbance at $490 \mathrm{~nm}$.

TUNEL-fluorescein isothiocyanate (FITC)/Hoechst 33258 apoptosis detection assay. Cell apoptosis was detected using a TUNEL-FITC Apoptosis Detection kit and a Hoechst Staining kit (Nanjing KeyGen Biotech Co., Ltd., Nanjing, China), according to the manufacturer's protocol. The A549-F10, A549-mock and untransfected A549 cells, treated with paclitaxel, were seeded at a density of $1 \times 10^{6}$ cells $/ \mathrm{ml}$ onto coverslips, washed three times (5 min each) with PBS, fixed in $4 \%$ formaldehyde for $20 \mathrm{~min}$, incubated in $70 \%$ ethanol at $-20^{\circ} \mathrm{C}$ for $30 \mathrm{~min}$, washed three times (5 min each) in PBS, and permeabilized in $0.1 \%$ Triton $\mathrm{X}-100 / 0.1 \%$ sodium citrate for $10 \mathrm{~min}$ at room temperature. Following another three 5-min washes with PBS, the cells were incubated with $3 \% \mathrm{H}_{2} \mathrm{O}_{2}$ for $10 \mathrm{~min}$ at room temperature, followed by three washes in PBS for 5 min each. The cells were then incubated with a terminal deoxynucleotidyl transferase enzyme (BBI Life Science Corporation, Hong Kong, China) in the dark at $37^{\circ} \mathrm{C}$ for $90 \mathrm{~min}$. Following two 2-min washes in PBS, the nuclei were stained with Hoechst 33258 in the dark at room temperature for $20 \mathrm{~min}$. Finally, the cells were washed in the dark three times in PBS containing 0.5\% Tween-20 for 2 min each, and were subsequently mounted in glycerol. The images were obtained using a fluorescence microscope (Nikon Corporation, Tokyo, Japan). The percentage of positive cells (as indicated by fluorescent staining) was calculated from the images.

Western blotting. The A549-F10, A549-mock and untransfected A549 cells were lysed using P0013 lysing agent (Beyotime Institute of Biotechnology, Suzhou, China), then cellular protein was separated by gel electrophoresis and transferred to membranes. The membranes were incubated with the anti-BAX, anti-caspase- 3 and anti- $\beta$-actin primary antibodies, followed by incubation with the horseradish peroxidase-labeled rabbit anti-mouse secondary antibodies. The blots were visualized using an enhanced chemiluminescence kit (Kodak, Rochester, NY, USA) and the signal intensities were quantified using SensiAnsys software (Shanghai Peiqing Science and Technology, Co., Ltd., Shanghai, China).

Statistical analysis. Data were analyzed using SPSS 13.0 statistical software (SPSS, Inc., Chicago, IL, USA) and presented as the mean \pm standard deviation. For the MTT cell proliferation assay, a factorial design analysis of variance (ANOVA) was used to determine any significant differences between the cell groups. The TUNEL-FITC/Hoechst 33258 assay and western blotting results were analyzed using a one-way ANOVA, followed by Fisher's least significant difference (LSD) post hoc tests when the variance was considered 
homogeneous, or by Welch's t-test and Dunnett's T3 test if the variance was considered heterogeneous. $\mathrm{P}<0.05$ was considered to indicate a statistically significant difference.

\section{Results}

Overexpression of F10 inhibits chemosensitivity to paclitaxel and paclitaxel-induced apoptosis in A549 lung cancer cells. To facilitate exploration of the underlying mechanisms of F10 in lung cancer, our previous study established a stable F10-overexpressing A549 cell line (13). The cell growth curve analysis revealed significantly increased cell growth in the F10-overexpression group compared with the control (13). In the present study, to determine whether the overexpression of F10 decreased the sensitivity of A549 lung cancer cells to chemotherapeutic agents, untransfected A549 cells, A549-mock cells and A549-F10 cells were treated with paclitaxel. An MTT assay was performed after $0.5,1,6,12,24$ or $48 \mathrm{~h}$ of paclitaxel treatment $(\mathrm{P}=0.621,0.036,0.486,<0.001$, $<0.001$ and $<0.001$, respectively; Fig. $1 \mathrm{~A})$. Concordant with the hypothesis, the proliferation of A549 cells was markedly inhibited in a time-dependent manner by paclitaxel: The difference between groups was significant $(\mathrm{F}=75.926 ; \mathrm{P}<0.001)$; the effect of time was significant $(\mathrm{F}=8.815 ; \mathrm{P}<0.001)$; and the interaction between group and time was also significant $(\mathrm{F}=8.815$; $\mathrm{P}<0.001)$. After $\geq 12 \mathrm{~h}$ of paclitaxel treatment, the proliferation rates of the cells in the three groups continuously decreased; however, in the A549-F10 cells, the paclitaxel-induced growth inhibition was significantly reduced compared with that in the two control groups (A549-mock and untransfected A549 cells; $\mathrm{P}<0.001)$. By contrast, proliferation was not observed to differ between A549-mock cells and untransfected A549 cells $(\mathrm{P}=0.126)$. Following treatment with paclitaxel for $48 \mathrm{~h}$, the growth inhibition rates of untransfected A549 cells, A549-mock cells and A549-F10 cells were 76.5, 80.4 and $64.2 \%$, respectively. The growth inhibition rate of A549-F10 cells following paclitaxel treatment was significantly lower than that in the A549-mock and untransfected A549 control cells $(\mathrm{P}<0.001)$. No difference in the growth inhibition rate was observed between the A549-mock cells and untransfected A549 cells ( $\mathrm{P}=0.201$; Fig. 1B).

The effect of F10 overexpression on paclitaxel-induced apoptosis was investigated. The untransfected A549 cells, A549-mock cells and A549-F10 cells were exposed to paclitaxel prior to double-staining with TUNEL and Hoechst 33258. Apoptosis was subsequently assessed according to the following criteria: Apoptotic cells were TUNEL-positive with nuclei exhibiting an intense blue with Hoechst staining; and normal cells were TUNEL-negative with nuclei exhibiting faint blue with Hoechst staining. In the merged images of the TUNEL and Hoechst 33258 staining, it was observed that the quantity of apoptotic cells in the A549-F10 group was markedly lower than in the two control groups (Fig. 2A). These results suggested that F10 overexpression inhibits paclitaxel-induced cell apoptosis in A549 cells. The TUNEL and Hoechst 33258 double-positive cells were counted in five randomly selected microscopic fields (magnification, x200) and the number of double-positive (apoptotic) cells following paclitaxel treatment was markedly different between the groups $(\mathrm{F}=200.778$; $\mathrm{P}<0.001$; Fig. 2B). A549-F10 cells exhibited markedly fewer
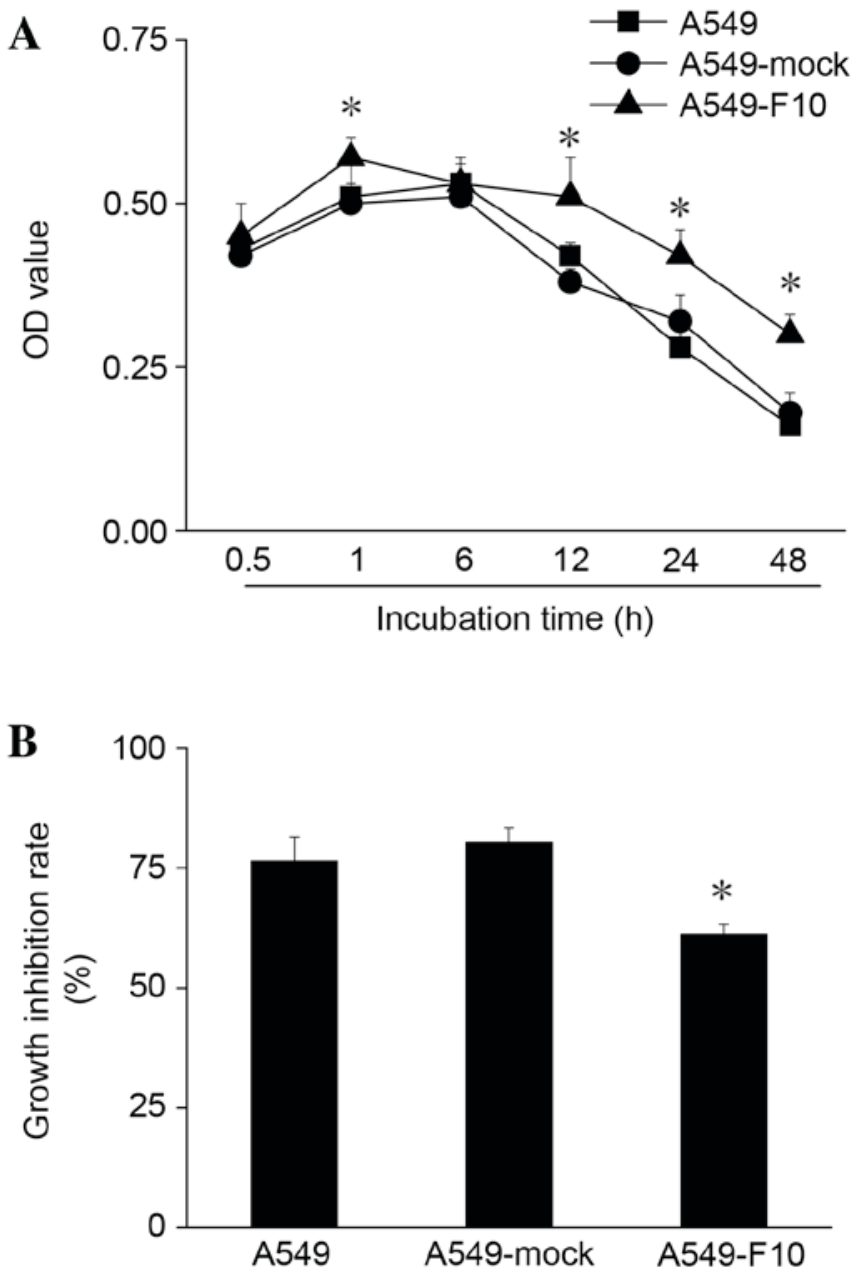

Figure 1. F10 decreases the anti-proliferative effects of paclitaxel in A549 cells. (A) The anti-proliferative effects of paclitaxel in various groups of A549 cells were determined by MTT cell proliferation assay. The growth inhibitory effects of paclitaxel in the A549-F10 cells were significantly lower than in the control groups of the A549-mock cells and the untransfected A549 cells $(n=6 ; P<0.05)$. No significant difference in proliferation was observed between the control groups ( $\mathrm{P}>0.05$ ). (B) Following $48-\mathrm{h}$ paclitaxel treatment, the growth inhibition rates of the untransfected A549 cells, A549-mock cells and A549-F10 cells were 76.5, 80.4 and 64.2\%, respectively. The growth inhibition rate of A549-F10 cells was significantly lower than in the two control groups $\left({ }^{*} \mathrm{P}<0.05\right)$. No significant difference in the growth inhibition rate was observed between the control groups $(\mathrm{P}>0.05)$. MTT, 3-(4,5-dimethylthiazol-2-yl)-2,5-diphenyltetrazolium bromide; OD, optical density.

apoptotic cells than A549-mock cells and untransfected A549 cells $(\mathrm{P}<0.001)$. However, the quantity of apoptotic cells was not observed to differ between the untransfected A549 cells and the A549-mock cells ( $\mathrm{P}=0.261)$.

These results indicate that the overexpression of F10 decreases the antiproliferative effects of paclitaxel and inhibits drug-induced apoptosis in lung cancer A549 cells. Therefore, the overexpression of F10 may desensitize certain types of lung cancer cells to chemotherapeutic agents.

F10 inhibits chemosensitivity to paclitaxel in A549 lung cancer cells by downregulating the pro-apoptotic genes $B A X$ and caspase-3. To study the mechanisms underlying the F10-mediated inhibition of paclitaxel chemosensitivity in A549 cells, the expression levels of BAX and caspase-3 
A
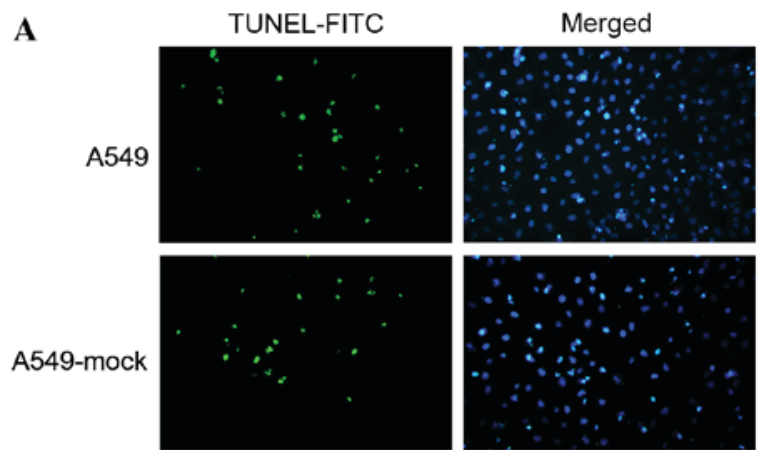

A549-F10
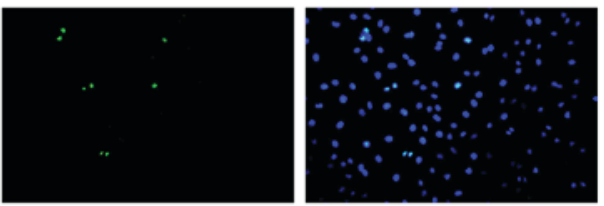

B

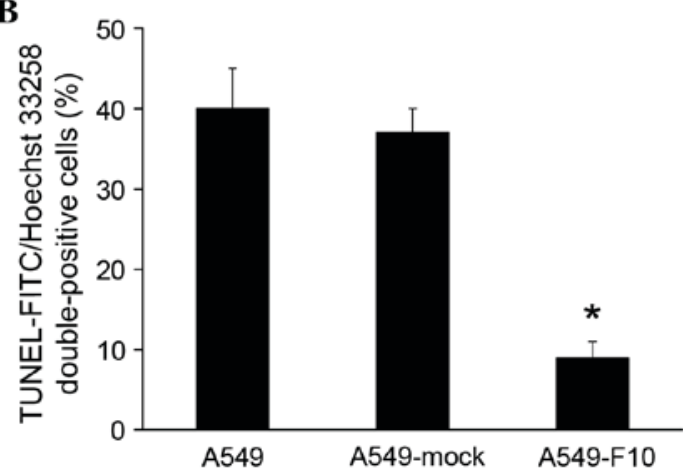

Figure 2. F10 inhibited paclitaxel-induced apoptosis in A549 cells. (A) Paclitaxel-treated untransfected A549 cells, A549-F10 cells and A549-mock cells were double-stained with TUNEL-FITC and Hoechst 33258 and observed under a fluorescent microscope. For each cell type, five fields (magnification, x200) were randomly selected, and the number of TUNEL-FITC (green) and Hoechst 33258 (blue) double-positive cells were counted. (B) The number of apoptotic cells was significantly decreased in the A549-F10 group, compared with the untransfected A549 cells and A549-mock cells ( $(\mathrm{P}<0.001)$. The number of apoptotic cells was not observed to differ between the untransfected A549 cells and A549-mock cells $(\mathrm{P}=0.261)$. FITC, fluorescein isothiocyanate.

were examined. Untransfected A549 cells, A549-mock cells and A549-F10 cells were treated with paclitaxel and a western blot analysis was performed (Fig. 3A). Quantification of the results indicated significant differences in the expression levels of BAX (Welch=166.892; $\mathrm{P}<0.001)$ and caspase $-3(\mathrm{~F}=51.231 ; \mathrm{P}<0.001)$ between the three treated A549 cell lines. Following paclitaxel treatment, the Dunnett's T3 test demonstrated that the A549-F10 cells exhibited lower expression levels of BAX than those observed in the untransfected A549 cells and the A549-mock cells ( $\mathrm{P}=0.006$; Fig. 3B). Furthermore, the LSD test indicated that caspase- 3 was reduced the most in A549-F10 cells $(\mathrm{P}<0.001$; Fig. 3C). However, no significant differences in the expression levels of BAX $(\mathrm{P}=0.176)$ or caspase-3 $(\mathrm{P}=0.25)$ were observed between the A549-mock cells and the untransfected A549 cells. The results of the current study indicate that the F10-mediated inhibition of A549 cell chemosensitivity to paclitaxel may be facilitated through the downregulation of BAX and caspase- 3 expression.
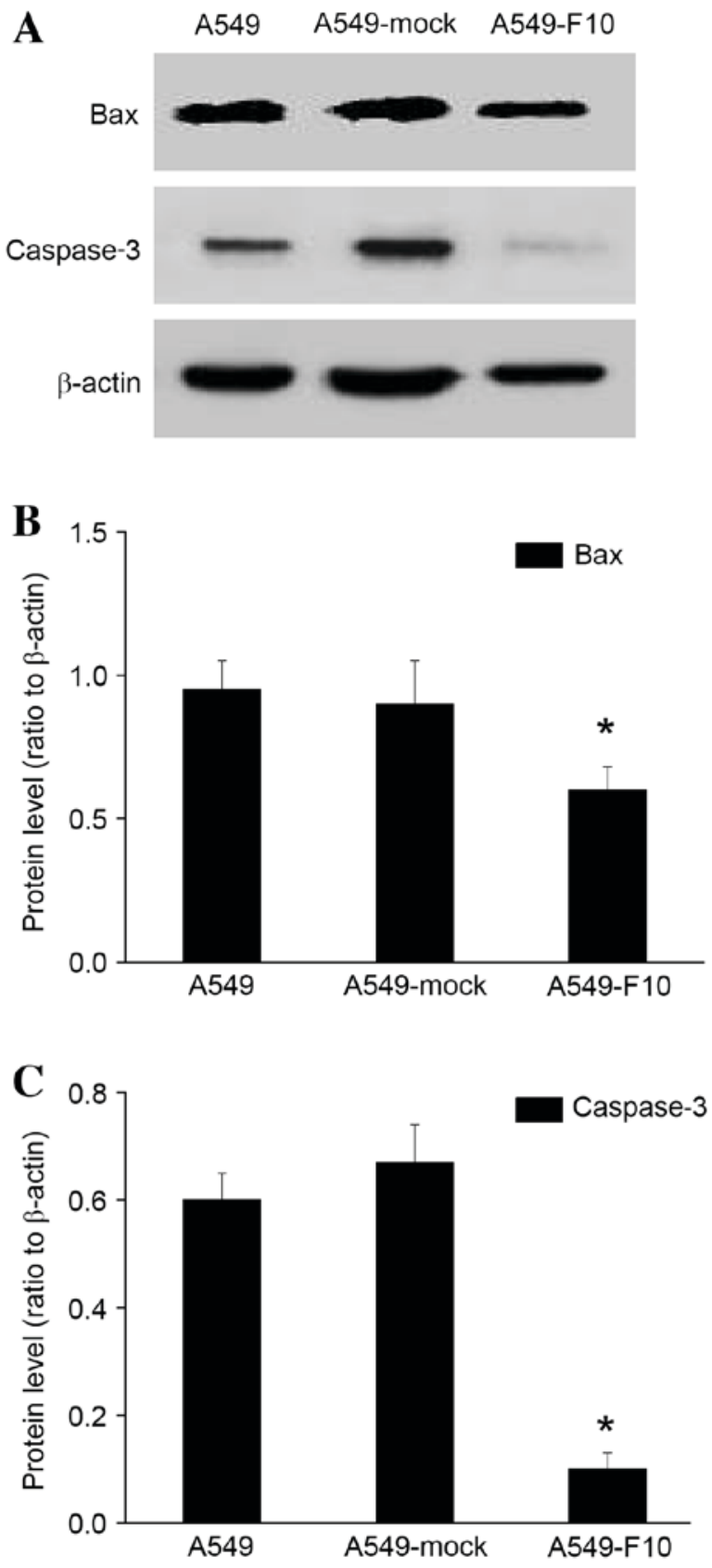

Figure 3. F10 reduced BAX and caspase-3 protein expression levels in paclitaxel-treated A549 cells. (A) The expression of BAX and caspase-3 was determined by a western blot analysis of paclitaxel-treated untransfected A549 cells, A549-mock cells or A549-F10 cells. The band signals of BAX and caspase-3 were normalized to that of $\beta$-actin. (B) The expression levels of BAX in paclitaxel-treated A549-F10 cells was lower compared with the untransfected A549 cells and A549-mock cells ( $\left.{ }^{*} \mathrm{P}<0.05\right)$. No significant difference in BAX expression levels was observed between the untransfected A549 cells and A549-mock cells $(\mathrm{P}=0.176)$. (C) The expression levels of caspase-3 in paclitaxel-treated A549-F10 cells were lower compared with the untransfected A549 cells and A549-mock cells ( $\left.{ }^{*} \mathrm{P}<0.05\right)$. The expression levels of caspase-3 were not observed to differ between the untransfected A549 cells and A549-mock cells $(\mathrm{P}=0.25)$. BAX, B-cell lymphoma-2-associated X protein.

\section{Discussion}

To elucidate the underlying mechanisms of the novel F10 gene in chemotherapeutic drug resistance, an F10-overexpressing A549 lung cancer cell line was established and paclitaxel was 
selected as a chemotherapeutic agent for the present study. The overexpression of F10 was observed to attenuate the antiproliferative effect of paclitaxel in A549 lung cancer cells. In addition, the overexpression of F10 was demonstrated to inhibit the paclitaxel-induced apoptosis of A549 cells. Finally, the results indicated that F10-mediated inhibition of paclitaxel chemosensitivity in A549 cells may be partially mediated through the downregulation of BAX and caspase-3 expression.

In our previous study, F10 was identified as a novel HM-associated gene that is expressed in a variety of adenocarcinomas $(10,11)$. Furthermore, F10 overexpression in A549 cells promoted cell proliferation in vitro and inhibited cell apoptosis $(12,13)$. However, the role of F10 in lung cancer chemoresistance had not been investigated. In the current study, it was identified that the overexpression of F10 decreased the antiproliferative effects of paclitaxel in A549 cells. Paclitaxel exhibits a high antitumor efficacy and is able to suppress cancer cell proliferation through the inhibition of mitosis (17). The results of the present study demonstrated that A549 cell proliferation was markedly inhibited, in a time-dependent manner, by paclitaxel, which is consistent with previous studies (18). However, the rate of paclitaxel-induced growth inhibition was markedly decreased in A549-F10 cells compared with the negative control, suggesting that the overexpression of F10 inhibited chemosensitivity to paclitaxel in A549 lung cancer cells.

Paclitaxel may exert antitumor effects by inducing tumor cell apoptosis $(2,17)$. Therefore, the present study investigated the association between F10 overexpression and the degree of paclitaxel-induced A549 cell apoptosis. TUNEL-FITC/Hoechst 33258 staining was used to determine cell apoptosis and revealed that, following paclitaxel treatment, the number of apoptotic cells in the A549-F10 group of cells was significantly lower compared with the controls. However, the degree of apoptosis was not observed to differ between A549-mock cells and untransfected A549 cells. This indicated that F10 overexpression could markedly inhibit paclitaxel-induced apoptosis in the A549 lung cancer cell line. Although these observations require further study for confirmation, the results presented support the hypothesis that F10 overexpression has an inhibitory effect on paclitaxel-induced apoptosis and paclitaxel chemosensitivity in the A549 lung cancer cell line.

Previous studies have identified that abnormal expression of the pro-apoptotic genes BAX and caspase-3 are associated with paclitaxel-induced cell apoptosis $(19,20)$. The current study also focused on the expression patterns of the BAX and caspase-3 in order to investigate the mechanisms underlying F10-mediated inhibition of paclitaxel chemosensitivity in A549 cells. The results revealed that the expression of BAX and caspase-3 in A549-F10 cells was significantly lower compared with the controls, indicating that F10 overexpression may alter the activity of BAX and caspase-3, affecting the sensitivity of A549 cells to chemotherapy. BAX and caspase-3 are pro-apoptotic factors and their direct activation is involved in apoptosis regulation. The expression of BAX and caspase-3 is positively associated with the apoptosis index (21). Although the underlying mechanisms require further investigation, the results of the present study indicate that the overexpression of F10 in A549 cells may lead to the downregulation of BAX and caspase- 3 expression, which may subsequently inhibit cell apoptosis. Therefore, this study may have provided experimental evidence to support the role of F10 in the inhibition of chemosensitivity to paclitaxel in certain lung cancer cells.

However, the current study had various limitations. Paclitaxel was used at a concentration of $10 \mathrm{nmol} / \mathrm{ml}$; higher doses have previously been reported to induce adverse effects in patients, including severe allergic reactions and myelosuppression, whereas lower doses have not previously been observed to effectively inhibit tumor growth (22). Therefore, it is necessary to identify the optimal concentration of paclitaxel, wherein the efficacy is maintained in a lower dose but the toxicity and adverse effects of higher doses are avoided. Further studies must employ a greater range of paclitaxel concentrations in order to determine the optimal dose and to increase the reliability of the results. Various cell lines must also be used to assess whether the observations of the present study may be confirmed in other lung cancer cell lines, and in other types of cancer.

In conclusion, the results of the present study demonstrate that the overexpression of F10 in A549 lung cancer cells decreases their chemosensitivity to paclitaxel through the downregulation of BAX and caspase-3 expression. The data support the value of F10 as a potential target gene for the chemotherapeutic treatment of malignancies characterized by high F10 expression, including ovarian cancer and endometrial carcinoma. Further studies must determine whether the overexpression of F10 is able to influence other types of cancer cells, chemotherapy drugs or anti-apoptotic factors, and whether the opposite effects would be exhibited following the knockdown of F10 in lung cancer.

\section{Acknowledgements}

The authors would like to thank Dr YG Cui, and Dr XM Cao (Department of Obstetrics and Gynecology, Southern Medical University, Guangzhou, China) for their technical assistance. This study was supported by the National Natural Science Foundation of China (grant no. 81401180), the Medical Scientific Research Foundation of Guangdong Province in China (grant no. A2013371) and the Population and Family Planning Commission Scientific Research Project of Guangdong Province in China (grant no. 20133058).

\section{References}

1. Liu HC, Chen GG, Vlantis AC, Tong MC and van Hasselt CA: Chemotherapy for laryngeal cancer-an apoptotic approach. Curr Drug Targets 9: 878-886, 2008.

2. Marupudi NI, Han JE, Li KW, Renard VM, Tyler BM and Brem H: Paclitaxel: A review of adverse toxicities and novel delivery strategies. Expert Opin Drug Saf 6: 609-621, 2007.

3. Khanna C, Rosenberg M and Vail DM: A review of paclitaxel and novel formulations including those suitable for use in dogs. J Vet Intern Med 29: 1006-1012, 2015.

4. Mori T, Kinoshita Y, Watanabe A, Yamaguchi T, Hosokawa K and Honjo H: Retention of paclitaxel in cancer cells for 1 week in vivo and in vitro. Cancer Chemother Pharmacol 58: 665-672, 2006.

5. Liu J, Meisner D, Kwong E, Wu XY and Johnston MR: Translymphatic chemotherapy by intrapleural placement of gelatin sponge containing biodegradable paclitaxel colloids controls lymphatic metastasis in lung cancer. Cancer Res 69: 1174-1181, 2009. 
6. Tien H, Dahlberg SE, Sandler AB, Brahmer JR, Schiller JH and Johnson DH: Prognostic models to predict survival in non-small cell lung cancer patients treated with first-line paclitaxel and carboplatin with or without bevacizumab. J Thorac Oncol 7: 1361-1368, 2012.

7. Ceresoli GL, Gregorc V, Cordio S, Bencardino KB, Schipani S, Cozzarini C, Bordonaro R and Villa E: Phase II study of weekly paclitaxel as second-line therapy in patients with advanced non-small cell lung cancer. Lung Cancer 44: 231-239, 2004.

8. Han ZX, Wang HM, Jiang G, Du XP, Gao XY and Pei DS: Overcoming paclitaxel resistance in lung cancer cells via dual inhibition of stathmin and Bcl-2. Cancer Biother Radiopharm 28: 398-405, 2013.

9. Li GT, Pang ZJ, Zhou J, Xing FQ, Li B, Chen SL and Fu X: Cloning of new genes associated with the pathogenesis of hydafidiform mole. Guangdong Medical Journal 27: 22-24, 2006

10. Zhou J, Chen SL, Xing FQ, Pang ZJ, Li B, Zhou WQ, Fu X and Ding YQ: Association of the novel hydatidiform mole-associated gene F10 with the invasiveness of trophoblastic tumor. Di Yi Jun Yi Da Xue Xue Bao 25: 171-173, 2005 (In Chinese).

11. Zhou J, Li B, Chen SL, Xing FQ, Pang ZJ, Zhou WQ, Fu X and Ding YQ: The expression of hydatidiform mole associated new gene F10 in different tumor tissues. Guangdong Medical Journal 26: 596-597, 2005.

12. Cao XM, Pang ZJ, Quan S and Xing FQ: Effect of F10 gene on expression of proliferating cell nuclear antigen and cyclin D1 Journal of Sun Yat-Sen University (Medical Sciences) 30: 6-9, 2009.

13. Song Y,Zhang G,ZhuX,Pang Z,Xing Fand Quan S:Overexpression of the hydatidiform mole-related gene F10 inhibits apoptosis in A549 cells through downregulation of BCL2-associated X protein and caspase-3. Oncol Lett 4: 419-422, 2012.

14. Song YL, Zhang G, Pang ZJ, Zhu XL, Yang XP, Li YF, Quan S and Xing FQ: Effects of gene F10 over-expression on the tumorigenicity of A549 cells. Med J Chin People's Liberation Army 37: 676-680, 2012
15. Song Y, Zhang G, Zhu X, Pang Z, Xing F and Quan S: Overexpression of the hydatidiform mole-related gene F10 inhibits apoptosis in A549 cells through downregulation of BCL2-associated X protein and caspase-3. Oncol Lett 4: 419-422, 2012.

16. Xiong HL, Zhou SW, Sun AH, He Y, Li J and Yuan X: Micro-RNA 197 reverses the drug resistance of fluorouracil induced SGC7901 cells by targeting mitogen activated protein kinase 1. Mol Med Rep 12: 5019-5025, 2015.

17. Symmans WF, Volm MD, Shapiro RL, Perkins AB, Kim AY Demaria S, Yee HT, McMullen H, Oratz R, Klein P, et al: Paclitaxel-induced apoptosis and mitotic arrest assessed by serial fine-needle aspiration: Implications for early prediction of breast cancer response to neoadjuvant treatment. Clin Cancer Res 6: 4610-4617, 2000

18. Xia RL, Lu Y, Zhu LN, Zhang SF, Zhao FK and Fu CY: Different regulatory pathways are involved in the proliferative inhibition of two types of leukemia cell lines induced by paclitaxel. Oncol Rep 30: 1853-1859, 2013.

19. Kim CH, Yoo JS, Lee CT, Kim YW, Han SK, Shim YS and Yoo CG: FHIT protein enhances paclitaxel-induced apoptosis in lung cancer cells. Int J Cancer 118: 1692-1698, 2006.

20. Woo IS, Jang HS, Eun SY, Kim HJ, Ham SA, Kim HJ, Lee JH, Chang KC, Kim JH, Han CW and Seo HG: Ran suppresses paclitaxel-induced apoptosis in human glioblastoma cells. Apoptosis 13: 1223-1231, 2008.

21. Mazumder S, Plesca D and Almasan A: Caspase-3 activation is a critical determinant of genotoxic stress-induced apoptosis. Methods Mol Biol 414: 13-21, 2008.

22. Peng C, Hao Y, Zhao Y, Sun Q, Zhao X and Cong B: Effect of Smac and Taxol on non-small-cell lung cancer. Acta Biochim Biophys Sin (Shanghai) 46: 387-393, 2014. 